\title{
Research on the Green Motivation of
}

\section{Knowledge-Based Employees}

\author{
Weidong Dai ${ }^{1}$ Xue Yang ${ }^{1}$ Lijiao Han ${ }^{1}$ \\ (Shenyang university of technology email-daiweidongll@163.com)
}

\begin{abstract}
This paper proposes an idea of "green motivation" by analyzing factors that impact knowledge-based employees of green motivation, and developing green motivation model. It is proposed that green motivation of the knowledge-based employees should be started on the employees' green needs, focused on accomplishing green material motivation and green psychological motivation. Green material motivation involves establishing a health incentive system, performing a flexible benefits system; while green psychological motivation involves pro-viding a healthy and safe occupational environment, implementing employee assistance programs and creating demo-cratic and harmonious organizational culture, to build a people-oriented moti-vation system for enterprises.
\end{abstract}

Keywords:green motivation; knowledgebased employees;green needs; sustainable development

The majority employees are in the "fatigue" state since the 1990s, and in 2006, a survey conducted by Fortune (Chinese version) which concluded that: most managers considered the pressure of work and life has brought many negative effects. It is estimated that the annual efficiency losses caused by employee health are more than 1 billion yuan ${ }^{[1]}$. Thus, paying attention to employees' physical and mental health, offering harmonious working environment beneficial to mental and physical pleasure, implementing green motivation will contribute to enterprise's successes in future competitions. In this case, researching on the green motivation is of profound theoretical and practical significance.

\section{Overview on the green motivation theory}

The researches on "green motivation" literature are relatively few. Wang Ping (2003), pointed out that the object of green motivation is knowledge-based employees, moreover, the green motivation should follow the law of all-round development of human, then make a effective promotion of enterprises in the changing market environment for sustainable development ${ }^{[2]}$. However, there is no definition of green motivation. He Xiaolian, Li Xiaocong have proposed the concept of green motivation in "The Green Motivation- The New Trend of Motivation Development" (2007).In their article, they deemed green motivation's premise of green person, and objective of meeting the employees' green needs, ultimately to actualize the motivation philosophy- employees' sustainable health. The premise of "green people" referred to the "self-actualizing man" developing into "green people" who pursues physical and mental harmony, health and happiness under theoretical hypothesis of Maslow's "Hierarchy of Needs Theory" and "Self-actualizing man". The core of green motivation sh-

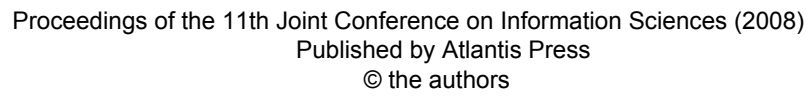


ould establish a motivation mode for managing work stress and keeping physical and mental health ${ }^{[3]}$. While these articles give an eye to the content and considered problems of green motivation. Based on the above-mentioned two studies, a new green motivation definition is given in this paper, what is more, taking knowledge-based employees' green needs as a breakthrough point, and then creating green motivation model with the approach of quantitative and qualitative analysis, accordingly, elaborating the green motivation.

\subsection{The definition of knowledge- based employees}

The concept of knowledge-based employyees originated from 'knowledge worker' which was being raised by Peter Diruke a managerial concepts' master. This definition referred to those who master symbols and concepts, utilize knowledge or information to work ${ }^{[4]}$. Specifically, knowledge-based employees who create wealth mainly live on the mental work; have master the world's latest science and technology; employ their knowledge and wisdom to add the highest degree of value to products; command the creation of wealth, are the main competitive advantage and a class participating the creation of surplus value for enterprise .

"Green" refers to the activities, behaviors, plans, ideas and some others on protection of the earth and ecological Specifically, the meaning of green includes two aspects: one is to create and protect the harmony ecological environ-ments in order to ensure human and economic sustainable development, the other is based on prohibited "red", and warning "yellow", thus "green" indicates the scientific, standardized and permanent unhindered actions ${ }^{[5]}$.

Needs are the core content of motivation. According to Herzberg's two-factor theory of motivation, "green needs" are divided into "green material needs" which have the hygiene factors to influence the employee pursues har-monious life and "green psychological needs" which have the motivator factors, furthermore, the employee eager to achieve a harmonious interpersonal re-lationships, psychological well-being and healthy emotion.

"Motivation" in the management concepts means adopt effective incentives to arouse the employee's initiative continuously, correspondingly combine the organization goals with the personal goals, so that the employee can be conducted to achieve maximum organizational goals.

To conclude, green motivations take the premise of harmonious development of human capitals, adhere to the "peopleoriented" principle, concern about the employees' green needs, fully arouse the employees' initiative and tap their potential to realize social and enterprise sustainable development. It is a considerable means to ensure organizational health, as well as a new motivation philosophy at the background of harmonious society. The sustainable development has two meanings here. First, maintaining the blood for full-scale development, during the pursue of self-survival and sustainable development, it is necessary not only to consider the business objectives of the market and improve the competitive position, but also to maintain the sus-tained growth on profits and abilities to raise in the leading competitive field and the intending expansion management environment, so that to make sure enterprise vigorous in a significant length of time, Its core is not the scale expansion, but to retain knowledge-based employees in order to adapt the environment and constant innovation. On the other hand, enterprises need to develop harmoniously with the external environment. It is said that the enterprise should give full considerations to develop coordinately with

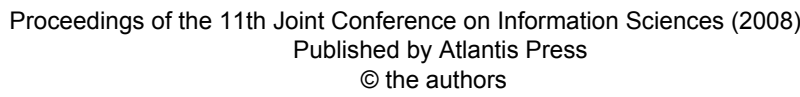


the environment, the society, and resources in pursuit of survival and pro-fits.

\section{The main problems of knowledge- based employees' green motivation}

Knowledge-based employees also have pressure caused by competition, which is from among project teams, the internal members of project and the whole field. Therefore, we need to help knowledgebased employee to correctly handle their pressure and relieve their feelings. Knowledge-based employees are the lifeblood of the development of enterprises. If they are misconducted, the enterprise will come up with incalculable losses.

The technical authority of knowledgebased employees is high, and their guide of technology in enterprises is great, too. But due to the characteristics of knowledge-based workers' work, their jobs are usually in a low administrative level. So if we don't carry out reasonable and effective methods of incentive mecha-nism, it is difficult to establish their au-thority and smooth their conduct work.

It seems that knowledge-based employees come to work and go home on time as others, but actually the time of their work is much more than normal working hours. Sometimes in order to maintain the continuity of thinking, they work on holidays or even sacrifice their sleeping. They use all their time to do the work.

\section{Creating the green motivation model}

\subsection{The ideology of green motivation model}

Japanese scholar Yilangin proposed a relational expression in Figure 3-1 which intuitively expressed the relationship between organizational goals and personal goals in the process of moti-vation .

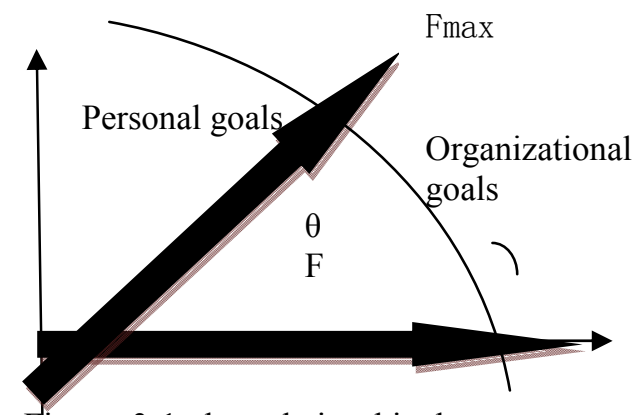

Figure 3-1 the relationship between organizational goals and personal goals.

Suppose $F$ max represents a person's maximum potential capacity; $F$ expresses a person's actual ability; $\theta$ indicates a correspondence between organizational goals and personal goals (included angle), so the relational expre-ssion is $F=F \max * \cos \theta\left(0{ }^{\circ}<\theta<90^{\circ}\right)$

It will be seen that the organizational and personal endeavor goals are in complete accord when $\theta=0^{\circ}, \cos \theta=1$, in the meantime, $F=F$ max , maximizing the use of the employee potential capacity to achieve the greatest effects ${ }^{[7]}$. The purpose is to encourage organizations to achieve the maximizing goal.

Therefore, in order to get the greatest satisfaction on green motivation, the enterprise's green motivation goal should be combined with the employees' green needs, consequently, the effectiveness would be maximized.

\section{2. developing the green motivation model}

Definition 1: supposing employees' (enterprises) green needs (the target) for the collection are

$\mathrm{Z}=\left\{\mathrm{Z}_{1}, \mathrm{Z}_{2}, \ldots, \mathrm{Z}_{n}\right\} \quad, \quad \mathrm{n}=1,2,3 \ldots$, through green motivation program $\lambda$, to meet the employees' green needs (the target) for the collection is 
$\beta=\lambda\left(Z_{1}, Z_{2}, \ldots, Z_{n}\right)=\left\{\beta_{1}, \beta_{2}, \ldots, \beta_{n}\right\}$

, and $\beta \in Z \quad, \quad \omega=\sum_{i=1} \beta_{i} / \sum_{j=1} Z_{j} \prec 1$

represent realizing part goals, or

$0<\omega=\frac{\sum(\beta i / Z i)}{n}<1$ expresses nume-

rous green needs (goals) are to achieve but the single goal is only be achieved partly, it can be considered the green motivation partly satisfied the employees green needs. At this time, $\omega$ is used to measure the degree of green motivation satisfaction.

Obviously, when $\omega=0$, the degree of satisfaction is zero; when $\omega=1$, it is fully satisfied.

Definition 2: Dividing knowledgebased employees' green needs into green material needs (including salaries, endowment insurance, medical insurance, free for regular medical check-ups, paid vocation, fitness award, and so on.) and green psychological needs (including the safe and comfortable working environment, good communication, emotional release, flexible working hours, harmonious interpersonal relationships, the update of knowledge, supporting, recognition of achievements, and so on.). Thus $\omega$ falls into $\omega_{1}, \omega_{2} . \omega_{1}$ indicates money, $\omega_{2}$ expresses with

$$
\omega=\sum_{i=1} \beta_{i} / \sum_{j=1} \mathrm{Z}_{j} \prec 1 \cdot
$$

Definition 3: Under the premise that the enterprises sustainable development goal are only relevant to the knowledgebased employee green motivation, the assumption is green material needs $\theta_{1}$, and green psychological needs $\theta_{2}$ which are known by survey, what is more, $\hat{\theta}_{1}, \hat{\theta}_{2}$ represent the satisfactions th-rough green motivation, so the degree of satisfaction is $\omega_{1}=\hat{\theta}_{1} / \theta_{1}, \omega_{2}=\hat{\theta}_{2} / \theta_{2}$.

Generally, enterprises' goals of sustainable development are simplified to the profits, and it is positive correlation with employee green motivation. Supposing the enterprises' profit is

$\eta\left(\omega_{1}, \omega_{2}\right)=\mathrm{A} \omega_{1}+\mathrm{B} \omega_{2}-C\left(\omega_{1}, \omega_{2}\right)+\varphi$, hereinto, $C\left(\omega_{1}, \omega_{2}\right)$ indicates the costs for satisfying knowledge-based employyees' green needs, it is relevant to $\omega_{1}, \omega_{2}$, and can be considered as a constant, because once the motivation perform is fixed, the costs are determined. Meanwhile, $\varphi$ is a influence factor. If the perform of green motivation make $\omega_{1}=\omega_{2}=1$, the goal of enterprise earnings will be fully realized

$$
\eta(1,1)=\mathrm{A}+\mathrm{B}-\mathrm{C}(1,1) \text {. }
$$

Therefore, the realization of enterprise's sustainable development goal is

$\omega=\eta\left(\omega_{1}, \omega_{2}\right) / \eta(1,1)=\frac{\mathrm{A} \omega_{1}+\mathrm{B} \omega_{2}-C\left(\omega_{1}, \omega_{2}\right)}{\mathrm{A}+\mathrm{B}-C(1,1)}$

If there is $\partial \omega / \partial \omega_{1}=\partial \omega / \partial \omega_{2}=0$, the degree of satisfaction on green motivation will be the maximum. In addition to

$$
\begin{gathered}
\partial \omega / \partial \omega_{1}=\frac{\mathrm{A}-C \omega_{1}}{\mathrm{~A}+\mathrm{B}-C(1,1)} \\
\partial \omega / \partial \omega_{2}=\frac{\mathrm{B}-C \omega_{2}}{\mathrm{~A}+\mathrm{B}-C_{(1,1)}}, \text { at the sa- } \\
\text { me time, } C \omega_{1}=\partial C^{\prime}\left(\omega_{1}, \omega_{2}\right) / \partial \omega_{1}, \\
C \omega_{2}=\partial C^{\prime}\left(\omega_{1}, \omega_{2}\right) / \partial \omega_{2}, \text { which can }
\end{gathered}
$$
be called as marginal satisfaction cost of green material incentives and psychological incentives.

Making the adjust factor of goal degree as $\alpha=\frac{\mathrm{A}-\mathrm{B}}{C \omega_{1}-C \omega_{2}}$. Therefore, the following conclusions are:

If $\alpha>1$, there will be

$$
\alpha=\frac{\mathrm{A}-\mathrm{B}}{C \omega_{1}-C \omega_{2}}>1 \text {. }
$$

That is to say,

$$
\begin{aligned}
& \frac{\mathrm{A}-C \omega_{1}}{\mathrm{~A}+\mathrm{B}-C(1,1)}>\frac{\mathrm{B}-C \omega_{2}}{\mathrm{~A}+\mathrm{B}-C(1,1)} \\
& \partial \omega / \partial \omega_{1}>\partial \omega / \partial \omega_{2}{ }^{[8]}
\end{aligned}
$$

The above shows the satisfaction of green material motivation made more contributions to enterprise sustainable development than green psychological mo- 
tivation, therefore, the implementation of green material motivation increase their profits more effectively. Similarly, the situation of $\alpha<1$ can be testified. If $\alpha=1$, the implementation of green material and psychological motivation will bring the same profits.

In addition, $C\left(\omega_{1}, \omega_{2}\right)$ is a constant that means costs of green motivation are fixed.

At this condition, if A > B, green material motivation makes more contributions to enterprise sustainable development than green psychological motivation, thus, green material motivation for knowledge- based employees will be better, otherwise is opposite.

If $\mathrm{A}=\mathrm{B}$, the implementation of green material and psychological motivation will bring the same profits, thus, no matter whether performing green material motivation or green psychological motivation, the results are the same.

From this, it can be found that the actualizing of green motivation goal are able to be tested by whether achieving the satisfaction of green motivation target in a certain period of time, if not, it is necessary to focus on the following as-pects to adjust their green material moti-vation and green psychological motiva-tion.

\subsection{Green material motivation}

Health award system refers to measures aiming at encouraging employee to enhance their own physical and mental health. Knowledge-based employees will be given special award when they are sensible of concerning about both physical and mental health. It's a method of guide employees to take the initiative to ease their own pressure.

Performing a flexible benefits system means making sure that the employee are able to decide their own combination of welfare program in a set time and within a fixed range of money. The benefits that they received will change with their life, which is a personalized and optional program of benefits.

\subsection{Green psychological motivation}

The building area, shape, ventilation, lighting and other physical factors are directly linked with employee's feelings, influencing their efficiency and satisfaction,so providing a safety occupational environment is extremely important.The employees' satisfaction and efficiency of jobs will be greatly reduced when they work in an unsuitable temperature and loud noises place ${ }^{[9]}$.

Employee assistance program provides advisory services for individuals and organizations in work place. It helps managers to identify the problems that the employee worries about. These problems may effect employee's working performance, or even affect the performance of the whole organization. The Advisory Council of experts will figure out plans to solve these problems. Employee assistance program services include personal life, work problems and organizational development. Some results showed that performing employee assistance program services effectively improve employee's work performance, job satisfaction and the commitment of organizations. In addition, it reduces the tendency of leaving the job.

The best reflect of organization values is the culture of organization. The right and scientific "green motivation" needs to be carried out with the open and democratic concept of organization culture. Employees need to be taken as assets, and their health and satisfactory are the strategic investment with commercial values. Employees need the chance of development and the support of society. Open and democratic organization atmosphere would promote good relationships within the organization. Furthermore, there will be a flat and non-centralized structure to support and encourage employees to par-

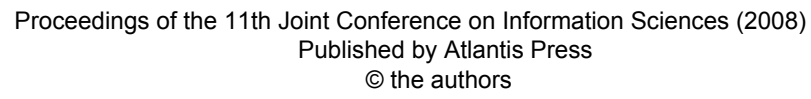


ticipate in the management, open channels of communication, encourage employees to meet the challenge, and promote the competition and cooperation among employees. We need to find the balance among meeting the customers' requirements, employees' needs, organization economic needs, and so on.

\section{Conclusion}

If the human capital as vital core competitiveness does not get effective motivation and protection, the development of enterprise will be threaten. Therefore, if enterprises would establish healthy organizations, they should focus on implementing green motivations on knowledge-based employees and meeting their green needs to achieve appropriate green motivations from both physical and psychological side. This paper simply analyses the linear model of green motivation. In fact, green motivation is a complex system. The satisfaction of green needs and the knowledge-based employees' performance will affect the development of enterprises. Therefore, the knowledge-based employees' performance evaluation will be the future direction of research.

\section{References}

[1] China's Wenzhou Chamber of Commerce network, "Concerned about Health Issues of Managers and Staffs"'[OL/D].Http://www.88088.co m.2006.12.2
[2] Wang Ping, "Reconstructing The Motivation Plantform- On Green Motivation Models And Green Modern Enterprises"[J]Technological Advancement Management, pp.137-138, 2003.12

[3] He Xiaolian, Li Xiaocong, "The Green Motivation- The New Trend of Motivation Development" [J] Technology Management Studies, pp135137, 2007 (01)

[4] Jiang Zhiqing, "Research on Knowledge-Based Employees' Moti-vation in China's State-Owned Enterprises"[D]. Harbin Engineering University, 2007.06

[5] Qiu Erwei, "Research on Green Management System" [D] Harbin Engineering University,.2006.

[6] Wang Dongmei, "Research on Motivation Mechanism of R\&D Staff in FAW Technology Center" [D]. Jilin University, 2005.12

[7] Li Baoyuan, "Strategic Motivation" [M]. Beijing: Economic Science Press, 2002.

[8] Peng Yunfei, "Research on The Contract Management for Enterprise Motivation Coupled with The Staff Needs

“[OL/D],http://www.paper.edu.cn.20 08.2 .

[9] Van Liere, Kent D. and Riley ED Dtmlap, "Environmental Concern: Doesn Make Difference How It Is Measured?" [J]. Environment and Behavior, pp. 652,2003 (6).

[10] Shi Kan, Zheng Rui, "Consideration of Healthy Organization Construction" [J]. Journal of.Capital Economic and Trade University, pp.13,2007(01). 